\title{
Redundant Array of Inexpensive Disks (RAID): Technology Description, Characterizations, Comparisons, Usage and Cost Benefits
}

\author{
Vish JUMANI \\ Boeing Computer Services, M/S: $7 \mathrm{~L}-10$ \\ P.O Box 24346 \\ Seattle, Wa 98126-0346
}

\begin{abstract}
:
Customer requirements have guided the evolution and implementation of software and hardware computer data storage technologies. The concept of RAID originated with a group of researchers at the University of California at Berkeley (UCB). They were investigating the use of an array of small capacity, inexpensive personal computer direct access storage devices (DASD) managed by a special type of a controller. as an alternative to the single large expensive disk (SLED) subsystems more common on mainframe computers. The disk arrays can be configured differently to provide different levels of operational capabilities, each providing special advantages in reliability and data availability, speed and economy. Different configurations are referred to as different levels of RAID. Berkeley originally defined a basic set of RAID levels ranging from RAID, level 0 (RAID 0) through RAID, level 5 (RAID 5). The RAID technology vendors have since extended the RAID level domain by defining RAID 6 and beyond to accommodate additional functionality. Each RAID type has shown itself to be suitable for certain applications. The projected reduction in storage costs and facilities, $\$ / G B$ and $\mathrm{ft}^{2} / \mathrm{GB}$ respectively, using the present RAID technology and its projected evolution are phenomenal.
\end{abstract}

\subsection{INTRODUCTION}

In this era of downsizing, distributed processing, high performance computing (HPC) environments and mainframe sites that rely on high availability and round-the-clock uptime, the old "glass house" disciplines that ensured data availability, reliability, and performance through intensive system management are no longer economically feasible. The need for continuous data availability, reliability, and performance in today's environment are greater than ever. The answer is to automate data availability and at the same time deliver improved reliability and performance. Today's applications running on high performance machines and mainframe sites require a high disk input/output $(\mathrm{I} / \mathrm{O})$ and a data storage architecture that will provide:

- Higher performance for a variety of applications, ranging from database to image processing - Higher availability of data and greater, more predictable application up-time - Flexible organization of data, and tools to better manage large volumes of data assets

- Configuration flexibility for a variety of customer environments

- Lower-cost storage solutions
In the interest of the reader, the following section reviews some of the major customer requirements that will continue to drive the evolution of the storage technology.

\subsection{STORAGE REQUIREMENTS}

Customer requirements over the years have directed the way in which hardware and software technologies have evolved and have been implemented in DASD storage subsystems environment. Hardware storage technology innovations have been used to vastly improve reliability, availability, performance, and capacity, as well as to greatly reduce the total cost of ownership. The following is a review of some major DASD storage subsystem requirements for today and for the future.

1. Availability - Important to customers because of increasing dependence upon round-the-clock, i.e., 24 hours/day, 7 days/week, $\left(24^{*} 7\right)$ operations. There are two major components of continuous availability: continuous operations and high availability.

2. Reliability - There is a need for the storage subsystem to help the user to maintain a continuous and reliable data source with a built-in capability to recover from component failures and errors and to keep the 
application and/or on-line user provided with the required data.

3. Performance - Important performance measures include: DASD response time, throughput, data transfer rate.

4. Support software - Host software to support new devices, data migration and management of data and integrated storage environments which will include DASD and other types of storage subsystems.

5. Modular Growth - Some important considerations here include: storage capacity and capacity increment.

6. Cost - The costs associated with storage include: cost/MB, cost per $1 / O$ s per sec, cost of redundancy, total cost - acquisition, maintenance, environmental, and residual.

7. Configuration fiexibility - The storage system must provide the flexibility to match capacity, performance, and availability needs of different business situations and application domains.

\subsection{THE STORAGE SOLUTION}

Armed with these requirements and responding to the rapidly improving processor technology as well as recognizing the limitation in absolute ability of the present DASD mechanical access mechanism to keep pace with the speed of the processors, a group of scientists at the UCB studied the potential use of disk arrays to address these requirements and to accommodate the technology improvements and limitations. The Berkeley hypothesis was that a collection of small inexpensive PC-type 5.25" and 3.5" disks (low cost/MB, low capacity, reliability and performance) connected together in certain logical ways could be made to deliver a high-capacity, low-cost storage solution with improved data availability, reliability, and performance relative to high-end $14^{\prime \prime}$ devices (high cost/MB, high capacity, reliability, and performance) common at mainframe levels through aggregating the data rates of several devices in the disk array. Higher data availability characteristics compared to high-end devices could be delivered through redundancy in the array. This disk array concept was labeled RAID. The scientists at the UCB defined six different levels of RAID, each implementing redundancy in a different manner effecting cost and other properties. The basic RAID sets defined are as follows: RAID 0 (Data Striping), RAID 1 (Mirroring), RAID 2
(Parallel Access, multiple parity devices), RADD 3 (Parallel Access, single parity device), RAID 4 (Independent Access, single parity device), and RAID 5 (Independent Access, distributed parity).

It has been seven years since the original RAID definitions were created. RAID has moved from concept to product domain. Since its first introduction, extensions to RAID have crept in as manufacturers have tried to optimize selected configurations from the original set of RAID for particular aspects.

\subsection{The Basic RAID Level Set}

The different RAID levels defined have characteristics which set them apart from one another. Some of them are very similar to each another except for the way the redundancy, parity storage, and recovery are implemented.

\subsubsection{RAID Level O (RAID 0)}

RAID 0 involves disk striping. User data is broken into chunks which is then stored on the striped set. It writes data across multiple drives and does not use parity or check disk. Spreading the data across drives allows simultaneous read and write operations and can result in performance improvement. It provides high $\mathrm{I} / \mathrm{O}$ because it transfers data to multiple drives at once. However, since RAID 0 does not write parity information, it is exposed to increased failure rate.

\subsubsection{RAID Level I (RAID 1)}

RAID 1 provides simple disk mirroring (or duplexing). The array's disk controller simultaneously writes data to two drives, but reads only from one. If the primary disk fails, the backup disk contains a copy of the current data. RAD 1 is, perhaps, the safest and fastest of RAID implementations. Duplication of disk resources is expensive on per-megabyte basis, but is unbeatable on throughput considering per dollar basis. Disk mirroring provides no check information and the system down time with mirrored images can be extensive.

\subsubsection{RAID Level 2 (RAID 2)}

RAID 2 introduces bit interleaving and check disks. When a block of data is written, the block is broken up and distributed (interleaved) across all of the data drives. At the same time, an error-correcting code (ECC) for the data block is also written, and spread across all of the check disks. This means that each write and read is performed simultaneously 
for all drives in the array. If a drive fails, the entire drive can be reconstructed from the distributed data and the check information.

\subsubsection{RAID Level 3 (RAID 3)}

RAID 3 is an improvement on RAID 2 but it is more complex. It replaces ECC with a parity bit for each byte written to disk, allowing such systems to use only a single check disk. User data is striped across all the data disks. Data chunks, which can be bits, bytes or blocks, are still interleaved across all data disks. Block sizes are set small with respect to request size. Under RAID 3, the drive spindles of all disks are synchronized, so the read/write heads pass over the same sector of the same track of each drive at the same time. This allows drives to run in parallel. RAID 3 is more reliable than the RAID 2 implementation and provides a more cost effective solution (See Figure 3.1.4-1).

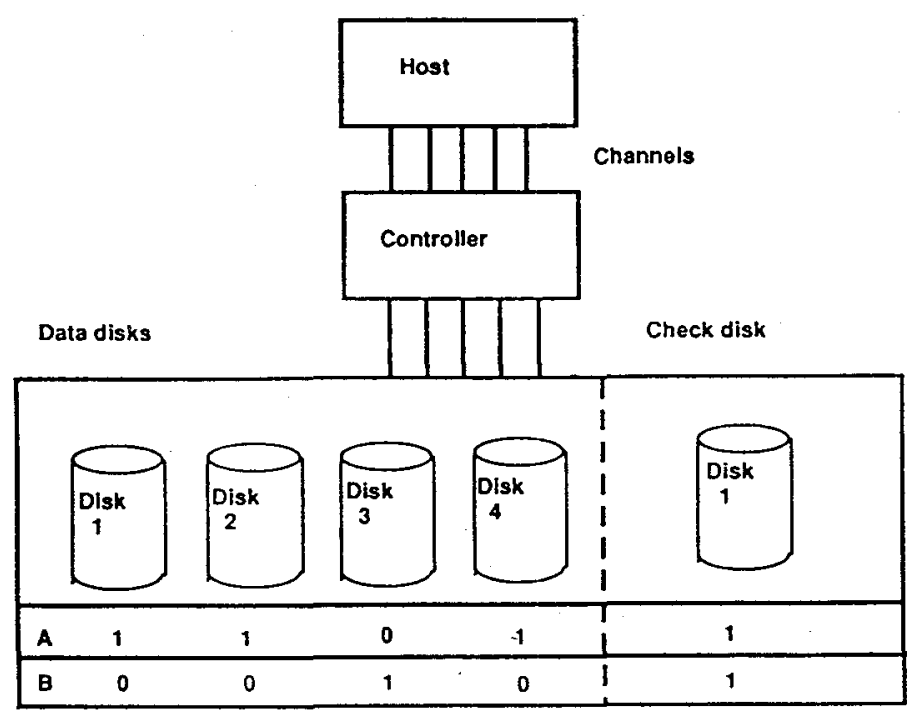

Figure 3.1.4-1 RAID Level 3: Data segments $A(1101)$ and $B(0010)$ are bitinterleaved across the four data disks. The check disk stores a parity bit for each group of data bits: in this case even parity ls enforced.

\subsubsection{RAID Level 4 (RAID 4)}

RAID 4 strips data on a sector level rather than the bit level as in RAID 3. Complete blocks of data are written to each drive in the array. As one sector of one drive fills, the next drive is addressed until its current sector is filled and so on across all of the drives. RAID 4 uses a parity drive and must write a sector of parity data each time it runs the gamut of the array drives. Each write involves reading, modifying, and writing the updated data and its updated parity setting. This slows the writing process. RAID 4 provides a heightened performance system involving large block reads and writes

\subsubsection{RAID Level 5 (RAID 5)}

RAID 5, like RAID 4, uses sector striping and can optionally include spindle synchronization. It does not use a separate check drive. It uses space on the existing array drives to record parity information (See Figure 3.1.6-1). Unlike RAID 3 however, RAID 5 allows for reads and writes to occur on separate disks independently and for simultaneous read and write operations to occur. It enables multiple reads and writes within a single logical drive and the system achieves a performance gain over RAID 3 because the parity information is written concurrently with the data. Because of the near equality of reading and writing speeds and other related qualities, RAID 5 is the most popular of the five RAID levels.

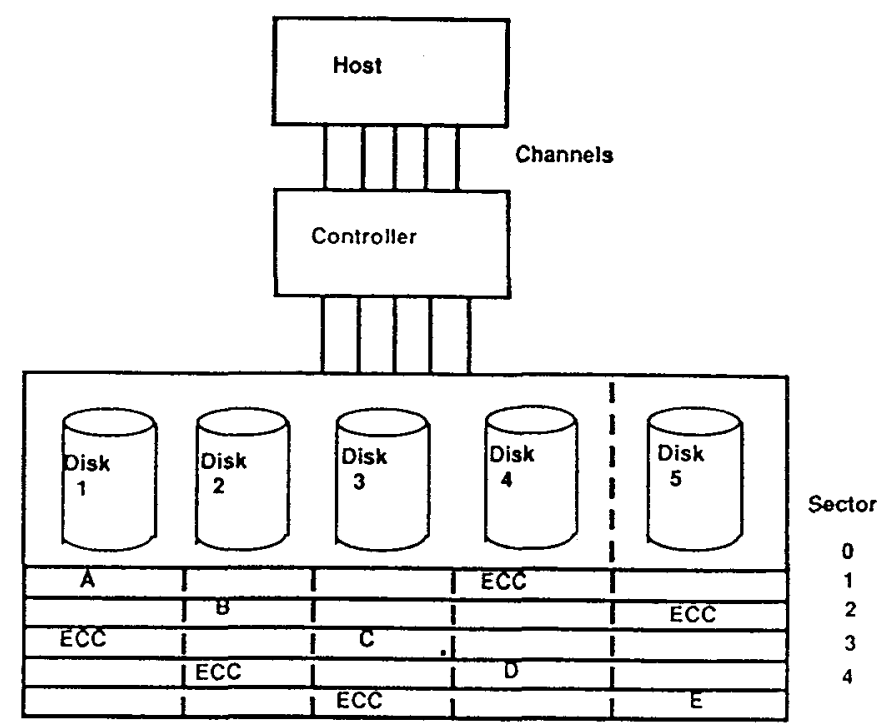

Figure 3.1.6-1 RAID Level 5: Dispersing ECC data throughout the system increases parallelism. Data segments $B$ and $C$ can be accessed at the same time; neither the segments nor their ECC data reside on the drives.

\subsection{RAID Extensions}

As mentioned before, variations have crept into the RAID environment as manufacturers have tried to optimize some 
aspect of the selected RAID configurations. Some have tried to optimize data transfer rates. Others have provided increased levels of data protection and availability. Still some others have introduced modifications to eliminate the RAID 5 write penalty. Some important extensions of RAID will be discussed below.

\subsubsection{RAID 6}

RAID 6 offers very high availability. Two drives are used for redundancy with sophisticated error-correcting codes. This capability enables RAID 6 to insure data integrity and availability even when two drives fail. Data and errorcorrecting information are striped across all members of the RAID set. Write performance is somewhat worse than RAID 5 , because three drives must be accessed twice during writes.

\subsubsection{RAID 10}

RAID 10 is actually RAID $0+1$. This is the combination of striping and mirroring, implemented by striping mirror sets. RAID 10 provides the best performance of any type of RAID by combining the performance advantages of RAID 0 and RAID 1. It also provides disaster tolerance. However, a write penalty is paid because both mirror-set members need to be written to.

\subsection{RAID Properties}

The RAID architecture primarily offers high storage capacities and dramatic increases in data availability. It also offers performance benefits depending upon the application.. The following sub-sections explain how the RAID concept and its built-in features aid data availability, performance, and cost.

\subsubsection{RAID and Availability}

Availability is usually the major benefit of RAID subsystems. In this context, availability is the ability of the system to continue accessing data despite a device failure. Availability is not a "yes" or a "no" choice. Actually, from a systems perspective, availability is a continuum. The steps one takes to enhance availability range from backing up a hard drive daily or once a week to maintaining disaster-tolerant systems with up-to-the-second data redundancy. For RAID, data redundancy is the key to high data availability. There are two major types of redundancy: mirroring and parity.

\subsubsection{Data redundancy: mirroring}

Mirroring is the simplest way to achieve data redundancy. For each data drive there is second drive that contains exactly the same data. Data is written to both drives. Thus, if one drive fails, the other drive can provide an exact copy of the lost data immediately. This process can be referred to as "mirroring" or "duplexing". In mirroring, there is one disk controller, and data is written serially, first to one drive and then to the mirror. This imposes a performance penalty. In duplexing, there are two controllers, and data is written to both drives in parallel, eliminating the performance penalty. The data availability of the mirrored or duplexed drives is excellent but costly.

\subsubsection{Data redundancy: parity}

The second way to provide data redundancy is to apply sophisticated mathematical coding techniques to produce parity data. Stored in the RAID array, parity data makes it possible to maintain access to data even if the physical drive on which the data was stored has failed. When there is a request to read data from the failed drive, the RAID automatically recreates the requested data from parity data stored on the other drives in the array. The advantage of parity is that it requires significantly less extra disk space than mirroring, because parity data for the whole array usually requires only one extra drive.

\subsubsection{Data reconstruction}

Data redundancy, whether it is based on mirroring or parity data, allows you to maintain active access to your data in spite of the drive failure. Reconstruction is the process of rebuilding data from the failed drive onto a replacement drive. During reconstruction, there will be a fall-off in system performance, because controller resources and Y/Os are being used to copy or reconstruct data using parity data. The speed of reconstruction depends on the available resources. This can be adjusted by the system manager. If transaction volume is high, the RAID software will allow for slow reconstruction and devote more time to transactions. Most recent RAID subsystems provide automated, on-the-fly reconstruction.

\subsubsection{RAID and Performance}

RAID cannot solve all performance problems. In fact, RAID's primary function has been data availability and/or fault tolerance operations. But the RAID architecture definition which incorporates concepts of disk arrays, data striping, data redundancy, multiple $\mathrm{I} / \mathrm{O}$ channels, and disk synchronization, makes it possible for different RAID levels 
to offer performance and availability benefits. There are other ways to improve performance that are as effective or more effective than RAID. One can meet the data integrity goals through programs for backup, security, and disaster recovery. But RAID is the best and probably the only way to dramatically improve availability.

\subsubsection{RAID and I/O performance}

The impact of $\mathrm{y} / \mathrm{O}$ workload on system performance depends on four basic elements: request rate, data transfer rate, read/write ratio, and hot spots. For most applications, the request rate is the limiting factor. General office automation, databases, transaction processing, and server applications tend to be request-rate intensive. In these applications, the number of requests is high, and the average request size tends to be small ( $<8 \mathrm{~KB}$ per request).

Some applications are limited by data transfer rate. Examples are CAD, imaging, and graphics. For these applications, request rate tends to be relatively low, but the average request size tends to be large $(>64 \mathrm{~KB})$. So the data rate requirement can be higher than a single drive can sustain, and the RAID with multiple drives working together can offer a big performance benefit.

Read/write ratio is important in evaluating RAID levels, because some RAID levels perform much better reading than writing. Read performance in RAID 1, RAID 5, and RAID 6 is considerably better than the write performance. So the read percentage can affect the response time one observes. Caching has an effect of reducing the total number of I/Os going to the disk. This improves the overall response times, but it also increases the percentage of writes. Under moderate I/O loads, this has little impact. However, under heavy loads when the total I/O activity gets close to saturating the RAID system, a high percentage of writes can degrade total $\mathrm{H} O \mathrm{O}$ performance. The fall-off in performance can be sudden, not gradual. If consistent performance is a requirement in the application, then the RAID subsystem should be. benchmarked and sized to meet the peak requirements, rather than the average requirements. Hot spots are largely a problem with high request-rate applications, since it is common for many requests to go to the same disk and overload that disk's request rate capacity.

\subsubsection{RAID data striping and performance}

The major source of RAID's performance benefit comes from data striping. Striping enhances performance by spreading data across multiple drives. Striping works by first breaking down user data into segments called "chunks". In a fivedrive RAID system (whose members may be called A, B, C, $D, E)$, the first chunk is placed on drive $A$, the second on $B$, the third on $C$, the fourth on $D$, and the fifth on $E$, and the sixth back on $A$ and the cycle being repeated. This goes on until all the user data is stored. The system administrator can set the chunk size based on the application requirements. The relationship between chunk size and average request size determines whether striping maximizes performance for request rate or data transfer rate. If the chunk size is set larger than the average request size, all of the drives may be able to service different $I / O$ requests simultaneously, thus significantly increasing request-rate performance.

If the chunk size is set smaller than the average request size, then multiple drives in the strip set can participate in a single request in parallel, thereby increasing the data transfer rate. This is most beneficial with large request sizes, for which data transfer time is a significant portion of the total access time.

Load balancing is an automatic outcome of the striping concept within RAID. Striping spreads the data among several drives, so the IOO workload is balanced across several drives, and the total system performance benefits. The load balancing effect of striping accounts for much of the observed performance benefit of RAID subsystems.

\subsubsection{RAID and Cost}

At present, RAID costs more than the conventional drives of equivalent capacity and some RAID levels cost more than others. If data availability is of primary concern and if anything less than $100 \%$ availability means lost revenue, then the premium paid for RAID should be well worth the investment. If the requirement is not that clear, then one needs to look at the cost of acquiring RAID, versus the cost of the loss of availability. The high data availability within RAID does not obviate the need for backup because RAID does not take into account human error and/or other major disasters.

The values, cost/GB and (storage area)/GB, are some of the many metrics that are being used by system integrators and data center personnel to evaluate the storage technologies in planning support for the present and future requirements. The projected reduction in storage costs realized by the application of the present RAID technology and its projected evolution are phenomenal. The savings will come through the reduced storage costs $(\$ / G B)$ due to higher density storage and compact media (e.g., 16GB on $31 / 2^{\prime \prime}$ dia. media 
or $10 \mathrm{~GB}$ on $11 / 2$ " media by the year 2000 ) and as a consequense the reduction of the storage footprint $\left(\mathrm{ft}^{2} / \mathrm{GB}\right.$ ) that is made possible through this compactification of the storage media. Figures 3.3.3-1 and 3.3.3-2 show the projected cost in $\$ / \mathrm{GB}$ and the storage footprint in $\mathrm{ft}^{2} / \mathrm{GB}$ for the RAID technology.

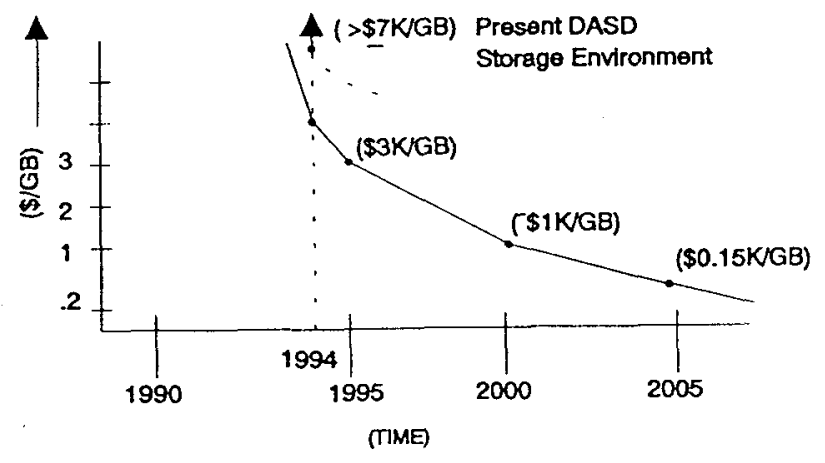

Figure 3.3.3-1: RAID \$/GB Projections (conservative) (On-line storage for large systems)

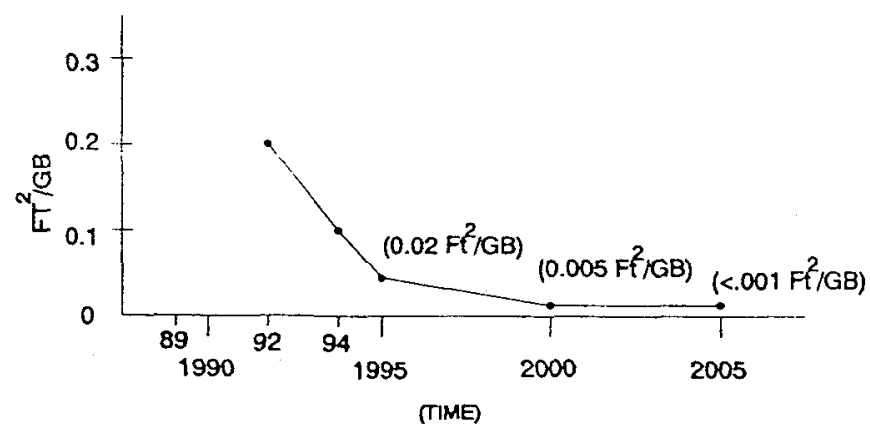

Figure 3.3.3-2: RAID FT' 4 GB Prolections (COTS - on-line storage for large systems)

\subsection{RAID Comparisions}

Figure 3.4 -1 provides a RAID level comparison. The chart gives a short description of each RAID level and rates each with respect to the other in terms of availability, read/write capability, cost, and type of application.

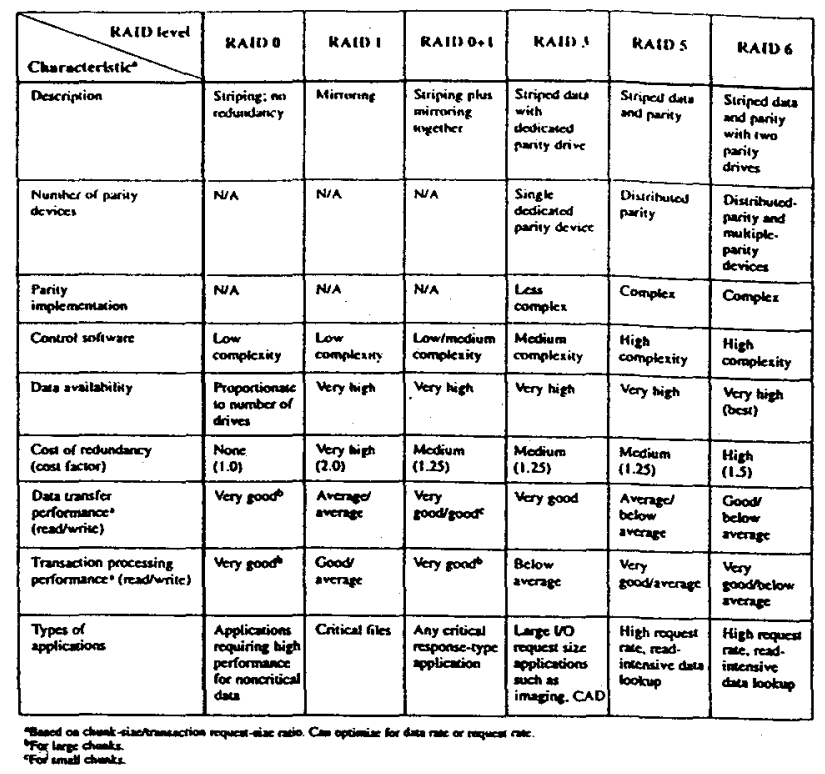

Figure 3.4-1. Raid Level Comparisons

\subsection{SUMMARY}

Mainframe computer centers and High Speed Computing (HPC) sites that rely on high availability, round-the-clock uptime, and performance can realize tremendous productivity improvements by implementing RAID. The RAID concept and technology provides a high capacity, reliable, high data availability and performance enhancing data storage environment with a configuration flexibility to support different types of applications and customer requirements. No single RAID level implementation can satisfy the needs of all customers. Different RAID levels represent a tradeoff between cost, availability, and performance. The projected reduction in storage costs and facilities, \$/GB and $\mathrm{ft}^{2} / \mathrm{GB}$ respectively, using the present RAID technology and its projected evolution are phenomenal.

\section{References:}

[1] Gary A. Coles, "Taking the Mystery out of Disk Arrays," Network Computing, October 1991.

[2] Steven J. Vaughn-Nichols, "Raiders of the Lost Disk," Computer Shopper, January, 1992.

[3] Paula Jacobs, "RAID: Better, faster or stronger," SunWorld, February, 1993.

[4] Digital Equipment Corporation, Storage Works Prespectives, "The Buyer's Guide to RAID Storage".

[5] S. J. Mckenna, IBM Corporation, Enterprise Systems Services and Support, DASD

Array, Washington Systems Center Technical Bulletin, Gaithersburg, Maryland. 